\title{
OBRA CIENTÍFICA E HONESTIDADE INTELECTUAL: UM COMENTÁRIO (E HOMENAGEM) À OBRA DE PEDRO PAULO ABREU FUNARI*
}

Víctor Revilla Calvo ${ }^{1}$

Como é habitual na comunidade científica, minha primeira aproximação com Pedro Paulo Abreu Funari foi indireta. Dito de outro modo, ele era para mim o que costumamos denominar como um "conhecido bibliográfico", alguém que conhecemos mais por sua produção científica do que pessoalmente. No caso de Pedro Paulo, esse conhecimento remonta ao período de fins da década de 1980, em que preparava minha tese de licenciatura sobre economia romana; seguida anos mais tarde por uma tese sobre o mesmo tema. Naquele momento, as indicações de meu diretor de tese me levaram a procurar e ler seus trabalhos científicos. Esses trabalhos me impactaram. Não se tratava apenas do fato de que a temática se movia pelo âmbito da economia romana e que, de algum modo, afetasse meu próprio trabalho. Neles haviam ideias, propunhamse hipóteses bem fundamentadas, desenvolvidas com um modo de pensar que ainda não era frequente na literatura científica espanhola. Por detrás havia também uma metodologia pouco habitual, determinada por uma perspectiva multidisciplinar, que recorria, sem inibições, à epigrafia sobre o instrumentum, à arqueologia e aos textos literários; estes últimos eram, além disso, desconstruídos cuidadosamente, uma vez que se percebia claramente sua condição de imagens codificadas e elaboradas pelas elites gregas e romanas.

Este conhecimento indireto converteu-se em relação pessoal na ocasião duma das várias visitas que Pedro Paulo realizou à Espanha; mais concretamente, como resultado de algumas de suas estadas para pesquisa em nossa universidade. Fruto dessa relação foi a proposta de realizar uma visita à Universidade Estadual de Campinas. Este projeto foi possível em 1999 graças ao auxílio da FAPESP. É difícil resumir as impressões dessa visita, determinante para mim tanto em nível científico

\footnotetext{
* Traduzido do espanhol por Rafael Aparecido Monpean, Doutorando em História, USP.

1 Professor Titular, Universidade de Barcelona, Barcelona, Espanha. E-mail: victorrevillac@gmail.com
} 
como pessoal, apesar de não ter sido muito longa. Graças a essa visita tive a oportunidade de conhecer outra comunidade acadêmica, outra prática

científica e, sobretudo, outra sociedade, a brasileira, construída sobre a base do multiculturalismo. Cheguei ao Brasil um pouco desorientado, sem saber nada mais do que tópicos sobre o país, que logo me mostrou toda sua diversidade. Também um pouco desorientado sobre o que eu poderia proporcionar ao currículo de uma universidade como a Unicamp. Podia interessar uma investigação centrada na história da conquista romana e a economia da Hispania? E, sobretudo, essa investigação podia conectar intelectualmente com as necessidades pessoais de alguns estudantes para os quais a História de Roma não constituía um legado cultural acriticamente aceito?

Sem dúvida, o balanço final foi extremamente positivo para mim. Em Campinas encontrei outra forma de organizar e de sentir a vida universitária, dentro de um quadro acadêmico que tinha pouco a ver com o da universidade espanhola: um quadro de convivência e atividade científica construído a partir de uma relação mais próxima e uma maior liberdade na relação professor-aluno, na troca de opiniões e na disposição; tudo isso construído a partir da constatação da forte diversidade de procedências e interesses dos membros da comunidade universitária. Tive a oportunidade, igualmente, de viver e conhecer outras localidades (em São Paulo, em Joinville), no âmbito universitário e na vida cultural dos museus locais e regionais, o que me permitiu apreciar as motivações que explicam a relação entre sociedade, ciência e patrimônio que inspiraram a ação e o pensamento de Pedro Paulo. Minha visita ao Brasil também me serviu para conhecer a alguns de seus alunos e discípulos. A relação com eles continuou nos anos posteriores, graças as suas visitas à Universidade de Barcelona. Todos eles demonstram a mesma motivação, liberdade de pensamento e curiosidade científica de seu mestre. O que é possível definir como a "marca de fábrica" de uma escola.

Este breve resumo de circunstâncias pessoais me serve como introdução para realizar um comentário mais específico sobre o que significou a influência da obra de Pedro Paulo em diversos campos da historiografia e da arqueologia da Antiguidade Clássica na Espanha.

A obra de Pedro Paulo Funari se desenvolveu em campos realmente muito diversos: desde os estudos mais específicos de cultura material da antiguidade até a arqueologia histórica, seguramente uma de suas áreas de trabalho preferidas. No caso de alguns pesquisadores esta diversificação corre o risco de conduzir à dispersão e à superficialidade. 
No caso de Pedro Paulo esta característica é o reflexo de uma profunda curiosidade intelectual que tem efeitos positivos, pois lhe permitiu aproveitar repetidamente as ideias e as práticas mentais desenvolvidas para uma área de pesquisa em outros campos. Bastará, nesse sentido, analisar o impacto do trabalho do autor em alguns campos de alta especialização.

O primeiro, o da cultura material romana, em que o autor dedicou uma atenção especial às evidências arqueológicas relacionadas com a produção e exportação de produtos alimentares na Roma antiga, em particular ao estudo da tipologia e da epigrafia das ânforas romanas como documento que permite a quantificação, a datação e a cartografia dos fluxos comerciais. Este estudo tem a vantagem adicional de oferecer a possibilidade de aprofundar o conhecimento da sociedade romana ao analisar fenômenos de consumo e gosto elaborados por classes e coletivos sociais diversos. Nas últimas décadas, a partir de 1960-1970, os estudos dedicados à cultura material da antiguidade e, em particular, às suas produções cerâmicas, experimentaram um desenvolvimento acelerado. Esse fenômeno foi traduzido numa autêntica explosão de publicações que, com extensão e profundidade diversas, abarcam todas as possibilidades de análise: desde monografias de escavação, nas quais se descrevem os repertórios associados às formas de vida e trabalho de um assentamento, até interesses regionais que abordam questões relacionadas com os mecanismos econômicos, culturais e políticos que impulsionaram a criação de alguns circuitos de troca de alcance mediterrânico. Mais recentemente, a partir da década de 1990, ao passo do desenvolvimento da informática, introduziu-se na História Antiga novos instrumentos de coleta, catalogação e análise da documentação que permitiu criar bases de dados cada vez mais amplas e complexas. Um exemplo disso é, dentre outros, a base de dados sobre epigrafia anfórica dirigida por J. Remesal, da Universidade de Barcelona, online desde 1991 (http://ceipac.ub.edu), ou a base sobre tipologia e arqueometria anfórica conduzida por S.J. Keay da Universidade de Southampton, Roman Amphorae: a digital resource, desde 2005)2. Estas bases de dados, que integram documentação tradicional (tipologia, epigrafia) e de novo tipo (arqueometria), proporcionam um impulso renovado no estudo da cultura material. Por exemplo, permitindo estabelecer relações entre

\footnotetext{
2 Podem ser citados outros recursos igualmente importantes, mesmo que com objetivos mais limitados: como a base de dados sobre ânforas de fabricação espanhola do Institut Català d'Arqueologia Clàssica de Catalunya (http://amphorae.icac.cat) ou o dedicado à arqueometria das ânforas das Gálias dos séculos I a III d.C., gerida pela Maison Archéologie \& Ethnologie, René-Ginouvès de Nanterre (www.mae.uparis10.fr/terresdamphores).
} 
diversos tipos de dados ou sequências cronológicas, assim como a análise estatística e a quantificação. Esta última possibilidade é imprescindível na história econômica, como visto no intenso debate teórico produzido nos últimos anos (Bowman, Wilson, 2009; Callataÿ, 2014).

Este interesse crescente pela cerâmica reflete a consciência da importância deste tipo de documentação para apresentar questões de caráter histórico: basta citar, como exemplo, o problema que supõe definir, em termos de continuidade e inovação, as mudanças que se produzem nas estruturas sociais ou econômicas e as mentalidades entre a República e o Império, para o qual a cultura material apresenta evidências importantes na forma de tradições artesanais (locais, regionais, provinciais) ou de novos hábitos de consumo e de trabalho (é o caso do fenômeno da reciclagem no âmbito doméstico). As novas questões, em chave histórica, que coloca esta evidência material tão modesta tem gerado uma renovação total de perspectivas e de abordagens de trabalho.

Os novos procedimentos analíticos, em particular, permitiu superar o recurso exclusivo à classificação tipológica como forma tradicional para identificar as produções e reconstruir os circuitos de trocas. De forma necessária, esta renovação conduziu à definição de espaços de debate cada vez mais delimitados pelos problemas tratados e pelos métodos utilizados. Seguindo a experiência desenvolvida no estudo da cultura material de outros períodos, os tipos de cerâmica distintos (especialmente as louças finas de mesa e as ânforas) são tratados cada vez mais como objetos de estudos específicos, que requerem uma aplicação particular dos procedimentos analíticos gerais e a elaboração de hipóteses apropriadas. Apenas desta forma é possível alcançar um conhecimento melhor das relações com outras manufaturas (entendendo-as globalmente como resultado de algumas tradições artesanais e culturais) e com a produção de outros bens para, em última instância, atingir a um maior conhecimento de certos aspectos da economia e das sociedades antigas. Toda a obra de Pedro Paulo Funari se integra nesse contexto de renovação teórica e metodológica, superando a perspectiva centrada na classificação tipológica de categorias particulares de louças ou de ânforas. É fundamental a este respeito a monografia redigida com C. Carreras sobre a circulação do azeite da Baetica ou Africa Proconsularis nas províncias do norte do Império Romano: Britannia y el Mediterráneo: estudios sobre el abastecimiento de aceite bético y africano en Britannia (Carreras Monfort, Funari, 1998).

Dedicar uma monografia ao estudo da distribuição e o consumo de um produto como o azeite não é o resultado de uma escolha arbitrária, e isto por várias razões. Em primeiro lugar, porque o azeite é, ao lado do vinho 
e dos cereais, um dos componentes da famosa tríade mediterrânica. Seu cultivo se associa a uma ecologia específica e a algumas condições de produção que as sociedades proto-históricas e históricas do mundo oriental e mediterrâneo desenvolveram e aperfeiçoaram ao longo de milênios. Trata-se, em outras palavras, de um recurso que simboliza um sistema socioeconômico e é, por sua vez, um elemento associado a processos de troca e contato cultural. O azeite é, por outro lado, um produto com usos muito diversos: alimento e condimento, combustível para iluminação, componente da higiene, da cosmética ou da medicina, etc. Seu uso, em grande quantidade e de forma regular, associa-se a um padrão de vida propriamente romano e depende, nas províncias do norte estudadas por Pedro Paulo, da existência de estruturas de comercialização. Mas este uso também depende da difusão e aceitação (ou não) de certos hábitos de consumo que supõem mudanças significativas nas práticas culinárias. Amostra disto são as diferentes formas de consumo nos assentamentos civis e militares da Bretanha e das Germânias que Pedro Paulo soube levar em consideração. Com esta percepção se adivinha o interesse pela história da cultura e das identidades culturais que constitui outro de seus campos de trabalho. $\mathrm{O}$ estudo de sua difusão, portanto, foi um meio de aprofundar o conhecimento do impacto da conquista romana sobre as sociedades celtas e germanas que ocuparam o espaço do continente europeu entre os séculos I a.C. e II-III d.C. Os trabalhos de Pedro Paulo, inseridos num diálogo enriquecedor com a obra de outros pesquisadores (por exemplo: Remesal, 1986, 1987; Whittacker, 1989), contribuiu na reconstrução das dinâmicas históricas geradas pela conquista romana no centro e no ocidente da Europa, situando a economia em seu lugar. Nessa mesma perspectiva se integra outras obras, como Cultura material e arqueologia histórica (Funari, 1998) e, sobretudo, Mercato. Le commerce dans les mondes grecs et romain (Funari, Pollini, 2012). Esta obra, escrita em colaboração com um dos maiores especialistas sobre finanças e bancos no mundo romano, Jean Andreau, contém uma seleção de textos literários clássicos que são analisados com um rigor profundo.

Outro dos campos de estudos ao qual Pedro Paulo se interessou é o que foi denominado comumente como História das mentalidades, cuja perspectiva é diferente à de uma História da Cultura (e ainda mais à de uma simples História das culturas). Não é necessário insistir em como os discursos literários dos autores antigos, membros de uma elite que pensava, atuava e imaginava a escala imperial, oferecem uma imagem da ordem social embasada na aceitação de uma hierarquia natural, com diferenças igualmente naturais de riqueza, status, e condição jurídica, sem falar das desigualdades sustentadas pelo gênero e pela idade, que tornam 
determinados coletivos invisíveis; esta invisibilidade afeta em especial a mulher, os grupos etários (os velhos ou as crianças) e os escravos. $\mathrm{O}$ estudo da identidade cultural é uma perspectiva que pretende dar voz a outros grupos, marginalizados por seu gênero, condição, status, pertencimento cultural, etc., rompendo com o sentido unidirecional, hegemônico e de autorrepresentação das elites de uma sociedade. A produção científica de Pedro Paulo, impossível de resumir aqui, é um exemplo de uma sensibilidade científica nova, que supõe o desenvolvimento de perspectivas novas, mas também de uma metodologia diferente, que analisa evidências novas ou já conhecidas.

O campo mais evidente é o dos graffiti. Esta prática é particularmente importante no mundo romano, como demonstram as inscrições parietais em Pompeia e em outros lugares da Campânia, assim como as milhares de inscrições em cerâmica recuperadas por todo império. Por meio desta evidência, que impactou fortemente a comunidade científica desde seu descobrimento no século XVII e que afetou o modo tradicional (quer acadêmico ou convencional) de avaliar a sociedade romana, foi possível se aproximar, de modo distinto, aos valores e aos comportamentos de coletividades marginalizadas pelo registro literário, definir os processos que conduzem à criação de formas de identidade coletiva e individual (social, cultural, de gênero, etc.) e analisar as relações entre os diversos grupos que integram uma sociedade tão fortemente hierarquizada como a romana; uma sociedade na qual, como resultado da conquista romana do Mediterrâneo, confluem uma cultura de elite (determinada por uma linguagem estética e alguns modelos éticos helênicos profundamente readaptados no período helenístico) e um mosaico de culturas regionais muito diversas. Estas culturas, por sua vez, foram mais ou menos influenciadas pelo legado grego desde a época arcaica: púnicos, etruscos, celtas, egípcios, persas. Estudar as manifestações escritas da sociedade romana, para além da literatura culta, significa desenvolver uma dupla perspectiva e uma dupla sensibilidade, ambas social e cultural. Só assim é possível tentar desentranhar os traços que caracterizam as diversas culturas locais no império ou a cultura popular romana (com limites imprecisos de definir) e sua evolução no tempo. De modo mais concreto, só a partir desta sensibilidade se pode perceber o processo de criação cultural por meio do qual coletivos definiram sua identidade e se situaram num contexto histórico complexo. $\mathrm{O}$ império romano, com a sua extensão e diversidade, é, nesse sentido, uma realidade muito complexa e difícil de captar.

Neste contexto histórico complexo, o uso da escrita em formas diversas, desde o signo até a palavra, em combinação com o uso de imagens, 
deveria contribuir para dar novos significados aos objetos cotidianos sobre os quais se escreviam, uma vez que lhes atribuía um valor comunicativo que relacionava indivíduos e certas iniciativas que se concentravam em um lugar. É isto que acontece com os milhares de objetos inscritos que a arqueologia recuperou, redigidos tanto em latim quanto em outras línguas que o império abrangia. Mas esta autêntica invasão da palavra escrita e da imagem é apreciada também em outro tipo de suporte: as paredes.

Este conjunto de práticas de comunicação se desenvolveu em lugares e situações precisas, relacionados com determinadas intenções ou determinadas funções que nem sempre podem ser especificadas ou explicitadas claramente. São mensagens cotidianas e imediatas, que respondem a necessidades de expressão imediatas e diretas; em outras palavras, são mensagens codificadas, cotidianas, sem pretensão de eternidade, difíceis de desentranhar. São, precisamente por essas razões, um meio fundamental de se aproximar das crenças e dos comportamentos reais de grande parte da população do império, uma vez que são partes de mecanismos de comunicação social e de definição de identidades desenvolvidas por indivíduos, coletivos pequenos e comunidades em situações e cenários diversos. Encontram-se dentre eles a religião, mas também os jogos complexos de alusões e de identificação pessoal criados pela convivência e o trabalho cotidiano que aparecem nas paredes ou sobre as louças. Não se pode excluir nenhuma dessas possibilidades. Conforme destacaram pesquisadores diversos, a definição das identidades na sociedade romana implicava processos ativos de seleção entre um repertório de imagens e uma cultura material, em contínua redefinição, produzida dentro de um quadro imperial; esta seleção era acompanhada por um uso preciso de formas diversas de mensagem escrita (Alfayé, Marco, 2008; Woolf, 1995; Hingley, 2005).

É neste campo de estudo em que se integra uma obra de formato pequeno, breve e simples, mas muito importante: La cultura popular en la antigüedad clásica (1991). Nela se reúne e interpreta um conjunto de exemplos de grafites parietais de Pompeia, caracterizados pela combinação de texto e imagem. Todos eles são conhecidos há muito tempo e numerosos pesquisadores destacaram, justamente, os temas que tratam esses grafites: situações relacionadas com o ambiente dos jogos de gladiadores (combates, heróis populares), o sexo, as fofocas do bairro ou da vida local. A análise feita destas evidências nesse livro soube destacar alguns princípios que caracterizam a linguagem popular e seus códigos: os jogos de palavras e as alusões mais ou menos claras, os exageros humorísticos, o uso cuidadoso dos insultos ou a palavra chula (nem 
sempre ofensiva) e de duplo sentido. Destacou-se também outro aspecto fundamental: a já aludida combinação de texto e imagem. Esta combinação é mais profunda do que pode parecer, uma vez que o texto não funciona simplesmente como uma ferramenta informativa. $\mathrm{O}$ texto adquire também um valor estético, supera os limites tradicionais da ordinatio, convertido em outra imagem. Deste modo, uma frase assume uma configuração sinuosa ou uma forma que sugere a mensagem transmitida: a feiura de uma pessoa por meio de características somáticas esquematizadas; a potência sexual, etc. A disposição do texto, em resumo, reforça e completa tudo que é representado pela imagem. São elementos imprescindíveis de uma mesma mensagem. A análise de Pedro Paulo, por meio de exemplos muito bem escolhidos, aprofunda esta e outras ideias para descobrir um código autêntico. Igualmente importante é a análise da estrutura dos textos e de seus elementos componentes, organizados em torno de uma série. Muitos desses aspectos foram ampliados em trabalhos posteriores (Funari, 1993a, esp. p. 169 e seguintes; 1993b).

A influência desta monografia é apreciada no trabalho de outros autores como N. Horsfall $(1996,2003)$, e pode ser percebida em trabalhos bem recentes, como a monografia de Kristina Milnor (2014) dedicada à Literary Landscape in Roman Pompeii. Poder-se-á argumentar que os grafites parietais das cidades do Vesúvio permitem apenas uma aproximação parcial e enviesada para o conhecimento da cultura das classes baixas da sociedade romana (uma visão "italocêntrica"). Mas existem indícios suficientes da extensão dessa prática por todo o Mediterrâneo, em todo tipo de situações e sobre todo tipo de suportes materiais (cerâmica, parede, pedra) para utilizar a evidência de Pompeia e Herculano como o meio mais direto para se aproximar de realidades e preocupações cotidianas de uma sociedade de dimensões imperiais. Utilizado uma expressão velha e feliz, também nessa esfera o período imperial supõe uma extensão do epigraphic habit. O trabalho de Pedro Paulo é completado com a exploração de linhas de investigação que abordam outras temáticas e recorrem a outros filões documentais. Pode-se citar a este respeito, Aspectos de La Cultura Popular Romana a Partir de Pompeya. Arte, erotismo y sensibilidad en el mundo romano (2012). Vários dos discípulos de Pedro Paulo desenvolveram esta linha de investigação com grande proveito. Basta recordar algumas das monografias de Renata Senna Garraffoni $(2002,2005)$ ou o trabalho de Lourdes Feitosa (2005).

Paralelamente, o trabalho de Pedro Paulo se insere em um campo mais amplo e que conheceu um desenvolvimento particular nas últimas décadas: a mudança cultural e os processos de definição das identidades 
em âmbitos diversos: social, religioso, político. O debate sobre isso é muito importante e foi iniciado com o questionamento radical de perspectivas (em primeiro lugar, a perspectiva colonial) e de terminologias, começando pela palavra "romanização". A bibliografia sobre o tema é enorme e o debate bem animado (Woolf, 1992, 1995, 1997, 1998; Webster, 2001; Hingley, 2005; Witscher, 2000; Terrenato, 1998, 2008, 2013). Neste cenário, os estudos pioneiros sobre cultura popular, um termo em si mesmo difícil de definir, adquirem um novo valor pela importância que concedem ao estudo integral dos grafites enquanto combinação específica de texto, imagem e suporte como um meio de definir as identidades individuais e coletivas na sociedade romana (Cooley, 2002; para a cultura popular na Roma imperial: Horsfall, 2003; Toner, 2009). A análise do autor ampliou-se nos últimos anos para a perspectiva de identidade, como demonstram algumas obras importantes e colóquios editados: Identidades, discurso e poder. Estudos de arqueologia contemporânea (2005), Politica e identidades no mundo antigo (2009), Identidades fluídas no judaísmo antigo e no cristianismo primitivo (2010). O tema da identidade é analisado nestes trabalhos a partir de perspectivas muito diversas (sociedade, religião, guerra, política) que relacionam mundo antigo e nossa época contemporânea.

Isso nos conduz até outro campo de estudo, de grande atualidade e que ultrapassa os limites estritos da pesquisa acadêmica: o da "recepção" do mundo clássico. O interesse pelos problemas em torno da transmissão do legado clássico, sua contribuição à definição de uma identidade (europeia ou ocidental) ou sua manipulação respondem, creio, a uma ideia particular: a "recepção" como um diálogo, em revisão contínua, entre nossa sociedade moderna e o passado. Este diálogo converte-se em debate quando se avalia o peso do legado clássico na composição da identidade brasileira contemporânea. A percepção da atualidade desse debate inspira iniciativas como a obra New Perspectives on the Ancient World: Modern perceptions, ancient representations (2008), editada por Pedro Paulo junto com R. S. Garraffoni e B. Letalien.

Por sua vez, a atividade de Pedro Paulo nesse campo inspira a elaboração de material didático e pedagógico, boa parte dos quais conheci e utilizei diretamente. Sua atividade se materializou na preparação de instrumentos de trabalho de tipo bem diverso. Estes instrumentos foram pensados para utilização em contextos muito diferentes, com atores igualmente diversos: o grande público, o entusiasta que deseja adentrar no mundo antigo, os jovens prestes a entrar na universidade. A elaboração desses tipos de instrumentos é especialmente difícil, uma vez que a simplificação (que implica reduzir uma informação em algumas 
poucas fórmulas redutivas) e a homogeneização excessivas devem ser evitadas por igual. Este duplo processo de simplificação e homogeneização encerra outro perigo: construir uma imagem do passado idealizada e que reproduz o discurso elaborado por determinadas elites, seja a sociedade escravista romana, seja o Brasil moderno e contemporâneo, uma sociedade também embasada parcialmente na escravidão - aspecto que já enfatizei anteriormente. A obra de P.P. Funari evita esse perigo e é consciente da necessidade de se dirigir a pessoas e coletivos cujos interesses e necessidades evoluem em função do contexto cotidiano em que atuam, como aficionados, como estudantes, como "consumidores" de cultura, etc.; os mesmos indivíduos, porém com interesses mutáveis. Felizmente, a comunidade científica está cada vez mais consciente da obrigação ética de difundir os resultados de uma atividade de pesquisa que tem sido sustentada pelo financiamento público e de divulgar esses resultados à sociedade. Uma obrigação ética, claro, mas também uma atuação guiada por fatores práticos: procurar o apoio social para uma prática científica cada vez mais custosa e cada vez mais difícil de justificar pela simples curiosidade científica. A obra de Pedro Paulo é pioneira nesse sentido, não apenas na sociedade brasileira, como também por sua influência no trabalho de muitos colegas em outros países do continente americano. Esse trabalho reúne uma ampla gama de matizes: Arqueologia (1988), A Cidade e a civilização romana: um instrumento didático (1997), Antiguidade clássica: a história e a cultura a partir dos documentos (2003), Patrimônio histórico e cultural (2006a), Patrimônio e cultura material (2006b), Arqueologia e patrimônio (2007).

É impossível fazer justiça ao valor da obra de Pedro Paulo ou, de modo mais simples, tentar resumir seu impacto no campo das ciências históricas, seja no Brasil, no campo acadêmico americano ou na Espanha. Se tivesse que explicar no que reside esse valor, teria que fazer referência, sem dúvida, à combinação de rigor científico, honestidade intelectual e sensibilidade. Uma combinação rara em nossa profissão, que tive o privilégio de conhecer pessoalmente.

\section{Bibliografia}

ALFAYÉ, S.; MARCO, F. Religion, language and identity in Hispania: Celtiberian and Lusitanian rock inscriptions. In: HÄUSSLER (ed.). Romanisation et épigraphie. Études interdisciplinaires sur l'acculturation et l'identité dans l'Empire romain. Montagnac: Éditions Monique Mergoil, 2008, 281-305. 
BOWMAN, A. K. \& WILSON, A. I. (eds.). Quantifying the Roman Economy: Methods and Problems. Oxford: Oxford University Press, 2009.

CALLATAY, F. de (ed.). Quantifying the Greco-Roman Economy and Beyond, Bari: Edipuglia, 2014.

CARRERAS MONFORT, C.; FUNARI, P.P.A. Britannia y el Mediterráneo: estudios sobre el abastecimiento de aceite bético y africano en Britannia. Barcelona: Edicions Universitat Barcelona, 1998.

COOLEY, A. (ed.). Becoming Roman, Writing Latin? Literacy and Epigraphy in the Roman West. Portsmouth, RI: Journal of Roman Archaeology, 2002.

FEITOSA, L.C. Amor e sexualidade: o masculino e o feminino em grafites de Pompeia. São Paulo: Annablume, 2005.

FUNARI, P.P.A. Arqueologia. São Paulo: Ática, 1988. 1991.

. La cultura popular en la Antiguedad Clásica. Écija: Editorial Sol,

El carácter popular de la caricatura pompeyana, Gerión, 11, 1993a, 153-173.

Graphic caricature and the ethos of ordinary people at Pompeii, Journal of European Archaeology, 1, 1993b, 133-150.

A cidade e a civilização romana: um instrumento didático. Campinas: Universidade Estadual de Campinas, Instituto de Filosofia e Ciências Humanas, 1997.

. Cultura material e arqueologia histórica. Campinas: Universidade Estadual de Campinas, Instituto de Filosofia e Ciências Humanas, 1998.

. Antiguidade clássica: a história e a cultura a partir dos documentos. Campinas: Unicamp, 2003.

. Patrimônio e Cultura Material. Campinas: Universidade Estadual de Campinas, Instituto de Filosofia e Ciências Humanas, 2006.

. Arqueologia e patrimônio. Erechim: Habilis, 2007.

Aspectos de la cultura popular romana a partir de Pompeya. Arte, erotismo y sensibilidad en el mundo romano. Barcelona: Editorial Académica Española, 2012. 
FUNARI, P.P.A.; ORSER JR, C.E.; SCHIAVETTO, S.N.O. Identidades, discurso e poder: estudos da arqueologia contemporânea. São Paulo: Annablume, 2005.

FUNARI, P.P.A.; PELEGRINI, S. Patrimônio Histórico e Cultural. Rio de Janeiro: Jorge Zahar Editor, 2006.

FUNARI, P.P.A.; GARRAFFONI, R.S.; LETALIEN, B.L. (eds.). New perspectives on the ancient world: modern perceptions, ancient representations. Oxford: Archaeopress, 2008.

FUNARI, P.P.A.; SILVA, M.A.O. (eds.). Política e identidades no mundo antigo. São Paulo: Annablume/Fapesp, 2009.

FUNARI, P.P.A.; NOGUEIRA, P.A. de S.; COLLINS, J.J. (org.). Identidades fluidas no judaísmo antigo e no cristianismo primitivo. São Paulo: Annablume/Fapesp, 2010.

GARRAFFONI, R.S. Bandidos e salteadores na Roma antiga. São Paulo: Annablume/FAPESP, 2002.

Gladiadores na Roma antiga: dos combates às paixões cotidianas.

São Paulo: Annablume, 2005.

HINGLEY, R. Globalizing Roman Culture: unity, diversity and empire, Londres: Routledge, 2005.

HORSFALL, N. M. La cultura della plebs romana, Barcelona: Promociones y Publicaciones Universitarias, 1996.

HORSFALL, N. M. 2003: The Culture of the Roman Plebs, Londres: Bristol Classical Press, 2003.

MATTINLGY, D. J. Imperialism, Power, and Identity. Experiencing the Roman Empire, Princeton: Princeton University Press, 2011.

MILNOR, K. Graffiti and the Literary Landscape in Roman Pompeii. Oxford: Oxford University Press, 2014.

REMESAL RODRÍGUEZ, J. La annona militaris y la exportación de aceite de oliva en Germania. Madrid: Editorial de la Universidad Complutense, 1986.

REMESAL RODRÍGUEZ, J. Heeresversorgung und die wirtschaftlichen Beziehungen zwischen der Baetica und Germanien. Materialien zu einem Corpus der in Deutschland veröffentlichten Stempel auf Amphoren der Form Dressel 20. Stuttgart: Kommissionsverlag Konrad Theiss, 1997. 
TERRENATO, N. The Romanization of Italy: global acculturation of cultural bricolage. In: FORCEY, C., HAWTHORNE, J., WITCHER, R. (eds.). TRAC 97. Proceedings of the Seventh Annual Theoretical Roman Archaeology Conference. Oxford: Oxbow Books, 1998, 20-27.

TERRENATO, N. The cultural implications of the Roman conquest. In: BISPHAM, E. (ed.). Roman Europe (the short Oxford history of Europe). Oxford: Oxford University Press, 2008, 234-264.

TERRENATO, N. Patterns of cultural change in Roman Italy. Non elitereligion and the defense of cultural self-subsistency. JEHNE, M., LINKE, B., RÜPKE, J. (eds.). Religiöse Vielfalt und soziale Integration. Die Bedeutung der Religion für die kulturelle identität und politische Stabilität im republikanischen Italien. Heidelberg: Verlag Antike, 2013, 43-60.

WEBSTER, J. Creolizing the roman provinces, American Journal of Archaeology, 105/2, 2001, 209-225.

WHITTAKER, C. R. Les frontières de l'Empire Romain. Besançon: Université de Franche-Comté, 1989.

WITCHER, R. Globalisation and roman identity: perspectives on identities in roman Italy. In: HERRING, E., LOMAS, K. (eds.). The Emergence of State Identities in Italy in the First Millennium BC. Londres: Accordia Research Institute, University of London, 2000, 213-225.

WOOLF, G. The unity and diversity of romanization, Journal of Roman Archaeology, 5, 1992, 349-352.

WOOLF, G. The Formation of Roman Provincial Cultures. In: METZLER, J. et alii (eds.). Integration in the Early Roman West. The role of Culture and Ideology. Luxemburgo: Musée National d'Histoire et d'Art, 1995, 9-18.

WOOLF, G. Beyond Romans and natives, World Archaeology 28/3, 1997, 339-350.

WOOLF, G. Becoming Roman: The Origins of Provincial Civilization in Gaul. Cambridge: Cambridge University Press, 1998. 